\title{
A normalized basis for reduced Clough-Tocher splines
}

\author{
Hendrik Speleers
}

Report TW546, August 2009

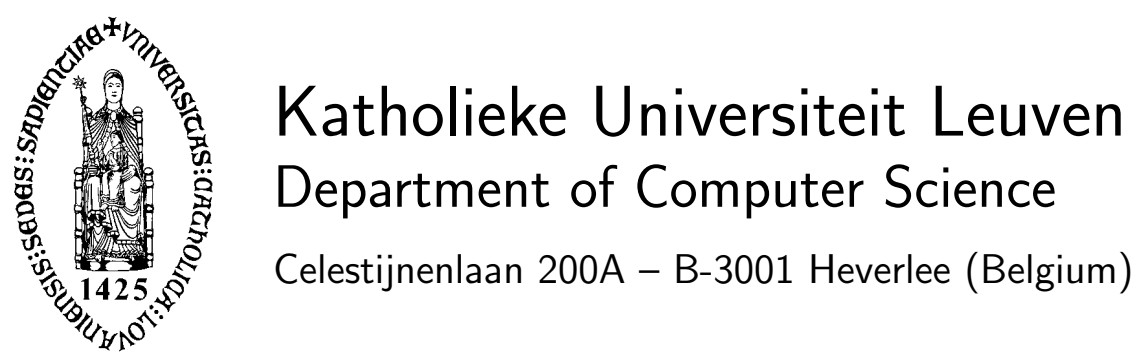




\title{
A normalized basis for reduced Clough-Tocher splines
}

\author{
Hendrik Speleers \\ Report TW546, August 2009 \\ Department of Computer Science, K.U.Leuven
}

\begin{abstract}
We present the construction of a suitable normalized B-spline representation for reduced Clough-Tocher splines. The basis functions have a local support, they are nonnegative, and they form a partition of unity. Geometrically, the problem can be interpreted as the determination of a set of triangles that must contain a specific set of points. This leads to a natural definition of tangent control triangles. We also consider a stable computation of the Bézier control net of the spline surface.
\end{abstract}

Keywords : Clough-Tocher splines, normalized B-splines, control points, control triangles, Bézier control net

MSC : Primary : 65D07, Secondary : 65D17, 68U07 


\title{
A normalized basis for reduced Clough-Tocher splines
}

\author{
Hendrik Speleers \\ Department of Computer Science, Katholieke Universiteit Leuven \\ Celestijnenlaan 200A, B-3001 Leuven, Belgium
}

\begin{abstract}
We present the construction of a suitable normalized B-spline representation for reduced Clough-Tocher splines. The basis functions have a local support, they are nonnegative, and they form a partition of unity. Geometrically, the problem can be interpreted as the determination of a set of triangles that must contain a specific set of points. This leads to a natural definition of tangent control triangles. We also consider a stable computation of the Bézier control net of the spline surface.
\end{abstract}

Keywords: Clough-Tocher splines, normalized B-splines, control points, control triangles, Bézier control net

AMS classification: 65D07, 65D17, 68U07

\section{Introduction}

A flexible mathematical representation is crucial for the geometric modelling of complex surfaces. A surface $s$ is usually represented as a linear combination of basis functions $\phi_{i}$,

$$
s=\sum_{i=1}^{N} c_{i} \phi_{i}
$$

where the basis functions satisfy certain properties. Typically they have a local support and form a convex partition of unity, i.e.,

$$
\phi_{i} \geq 0, \quad \text { and } \quad \sum_{i=1}^{N} \phi_{i}=1 .
$$

Continuity conditions can be imposed to obtain smooth surfaces.

The tensor product B-spline representation is very common in many computer aided geometric design packages [8]. With the B-spline control net surfaces can be locally adapted in an intuitive and flexible way. Piecewise polynomials defined on triangulations are an attractive alternative. PowellSabin splines can be represented in a normalized basis [4], in the sense that the basis functions form a convex partition of unity. They have an intuitive geometric interpretation involving control triangles. These splines are effective in a wide range of application domains (see, e.g., $[9,11,13,14]$ ). In this paper we consider Clough-Tocher splines. They were developed by Clough and Tocher [3] as a tool for the finite element method. Later on, they were also used in the area of scattered data interpolation $[6,10,12]$. The space of reduced Clough-Tocher splines is a particular subspace of the Clough-Tocher spline space. We will construct a compact normalized basis for this space. 
The paper is organized as follows. In Section 2 we review some general concepts of polynomials on triangles, and give the definition of the reduced Clough-Tocher spline space. Section 3 covers the construction of a normalized B-spline basis and presents a geometric interpretation: we are looking for a set of triangles that contain a specific set of points. In Section 4 we define a set of control triangles that are tangent to the spline surface. We also give a stable way to compute the Bézier ordinates of the spline, and briefly discuss the extension to parametric surfaces. Finally, in Section 5 we end with some concluding remarks.

\section{Reduced Clough-Tocher splines}

\subsection{Bivariate polynomials in Bernstein-Bézier representation}

Let $\mathcal{T}\left(V_{1}, V_{2}, V_{3}\right)$ be a non-degenerated triangle. Any point $P$ in the plane of the triangle can be uniquely expressed in terms of the barycentric coordinates $\tau=\left(\tau_{1}, \tau_{2}, \tau_{3}\right)$ with respect to $\mathcal{T}$, such that

$$
P=\sum_{i=1}^{3} \tau_{i} V_{i}, \quad \text { and } \quad \tau_{1}+\tau_{2}+\tau_{3}=1 .
$$

Let $\Pi_{d}$ denote the linear space of bivariate polynomials of total degree less than or equal to $d$. Any polynomial $p_{d} \in \Pi_{d}$ on triangle $\mathcal{T}$ has a unique Bernstein-Bézier representation

$$
p_{d}(\tau)=\sum_{i+j+k=d} b_{i j k} B_{i j k}^{d}(\tau),
$$

with

$$
B_{i j k}^{d}(\tau)=\frac{d !}{i ! j ! k !} \tau_{1}^{i} \tau_{2}{ }^{j} \tau_{3}{ }^{k}
$$

the Bernstein polynomials of degree $d$, which form a convex partition of unity on $\mathcal{T}$. The coefficients $b_{i j k}$ are called Bézier ordinates, and the Bézier domain points $\xi_{i j k}$ are defined as the points with barycentric coordinates $\left(\frac{i}{d}, \frac{j}{d}, \frac{k}{d}\right)$. By associating each Bézier ordinate $b_{i j k}$ with the Bézier domain points $\xi_{i j k}$, we can display the Bernstein-Bézier representation schematically as in Figure 1 for the case $d=3$. The piecewise linear interpolant of the Bézier control points, defined as $\mathbf{b}_{i j k}=$ $\left(\xi_{i j k}, b_{i j k}\right)$, is called the Bézier control net. This control net is tangent to the polynomial surface at the three vertices [7].

\subsection{The RCT-spline space}

Consider a simply connected subset $\Omega \subset \mathbb{R}^{2}$ with polygonal boundary $\partial \Omega$. Let $\Delta$ be a conforming triangulation $\Delta$ of $\Omega$, i.e., no triangle contains a vertex different from its own three vertices. Let $n, t$ and $e$ be the number of vertices, triangles and edges in $\Delta$, respectively. The vertices $V_{i}$ have Cartesian coordinates $\left(x_{i}, y_{i}\right)$.

The Clough-Tocher (CT-) refinement $\Delta^{*}$ of $\Delta$ partitions all triangles in $\Delta$ into three smaller triangles [3]. For each triangle $\mathcal{T}$, a point $Z$ is chosen in the interior of $\mathcal{T}$ and it is connected to the three vertices of $\mathcal{T}$ by straight lines. In this paper, we will restrict the position of these split points such that the line joining the split points of adjoining triangles $\mathcal{T}_{i}$ and $\mathcal{T}_{j}$ intersects the common edge of $\mathcal{T}_{i}$ and $\mathcal{T}_{j}$. Note that this is equivalent to the restriction on the split points used in defining Powell-Sabin refinements (see, e.g., [4]). Choosing each split point as the incentre of its triangle satisfies this restriction, but other choices may be more appropriate from a practical point of view. 


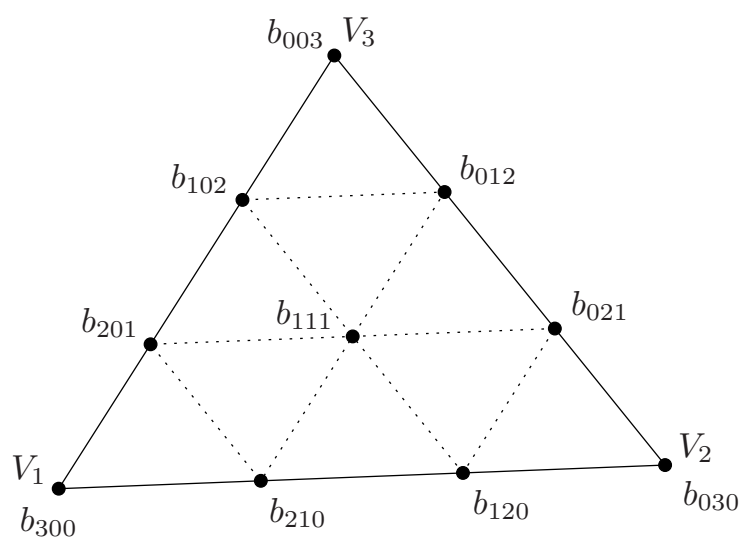

Figure 1: Schematic representation of the Bézier ordinates of a cubic bivariate polynomial.

The space of piecewise cubic polynomials on $\Delta^{*}$ with global $C^{1}$-continuity is called the CloughTocher spline space, i.e.,

$$
S_{3}^{1}\left(\Delta^{*}\right)=\left\{s \in C^{1}(\Omega):\left.s\right|_{\mathcal{T}^{*}} \in \Pi_{3}, \mathcal{T}^{*} \in \Delta^{*}\right\} .
$$

The dimension of the space equals $3 n+e$. Each of the $3 t$ triangles resulting from the CT-refinement is the domain triangle of a cubic Bernstein-Bézier polynomial.

We now consider a particular subspace of $S_{3}^{1}\left(\Delta^{*}\right)$. For each interior edge $\varepsilon$ of $\Delta$, let $\nu_{\varepsilon}$ be a unit vector parallel to the line joining the split points of the two triangles sharing $\varepsilon$. For every boundary edge $\varepsilon$ of $\Delta$, let $\nu_{\varepsilon}$ be a unit vector parallel to the line joining the midpoint of $\varepsilon$ and the split point of the triangle containing $\varepsilon$. The reduced Clough-Tocher (RCT-) spline space is defined as

$$
\hat{S}_{3}^{1}\left(\Delta^{*}\right)=\left\{s \in C^{1}(\Omega):\left.s\right|_{\mathcal{T}^{*}} \in \Pi_{3}, \mathcal{T}^{*} \in \Delta^{*} ;\left.\frac{\partial s}{\partial \nu_{\varepsilon}}\right|_{\varepsilon} \in \Pi_{1}, \varepsilon \in \mathcal{E}\right\},
$$

with $\mathcal{E}$ the set of edges of $\Delta$. The dimension of this space equals $3 n$. The following Hermite interpolation problem has a unique solution $s(x, y) \in \hat{S}_{3}^{1}\left(\Delta^{*}\right)$ for any given set of $\left(f_{k}, f_{x, k}, f_{y, k}\right)$ values with $k=1, \ldots, n$ :

$$
s\left(V_{k}\right)=f_{k}, \quad \frac{\partial s}{\partial x}\left(V_{k}\right)=f_{x, k}, \quad \frac{\partial s}{\partial y}\left(V_{k}\right)=f_{y, k}, \quad k=1, \ldots, n .
$$

Note that $\hat{S}_{3}^{1}\left(\Delta^{*}\right)$ is defined slightly different from the reduced Hsieh-Clough-Tocher spline space described in $[1,2,12]$. There, the derivative of the spline along an edge in the direction normal to that edge is restricted to be a linear polynomial.

\subsection{A Bernstein-Bézier representation for RCT-splines}

We consider a single macro-triangle $\mathcal{T}\left(V_{1}, V_{2}, V_{3}\right)$ in $\Delta$ with CT-split point $Z$, as indicated in Figure 2. When edge $V_{i}-V_{j}$ is a boundary edge, point $R_{i j}$ is taken to be the midpoint of the edge, otherwise $R_{i j}$ is the intersection point of the edge and the line joining the split points of the two triangles sharing the edge. We assume that the points indicated in the figure have the following barycentric coordinates:

$$
\begin{aligned}
& V_{1}=(1,0,0), \quad V_{2}=(0,1,0), \quad V_{3}=(0,0,1), \quad Z=\left(z_{1}, z_{2}, z_{3}\right), \\
& R_{12}=\left(\lambda_{12}, \lambda_{21}, 0\right), \quad R_{23}=\left(0, \lambda_{23}, \lambda_{32}\right), \quad R_{31}=\left(\lambda_{13}, 0, \lambda_{31}\right) .
\end{aligned}
$$




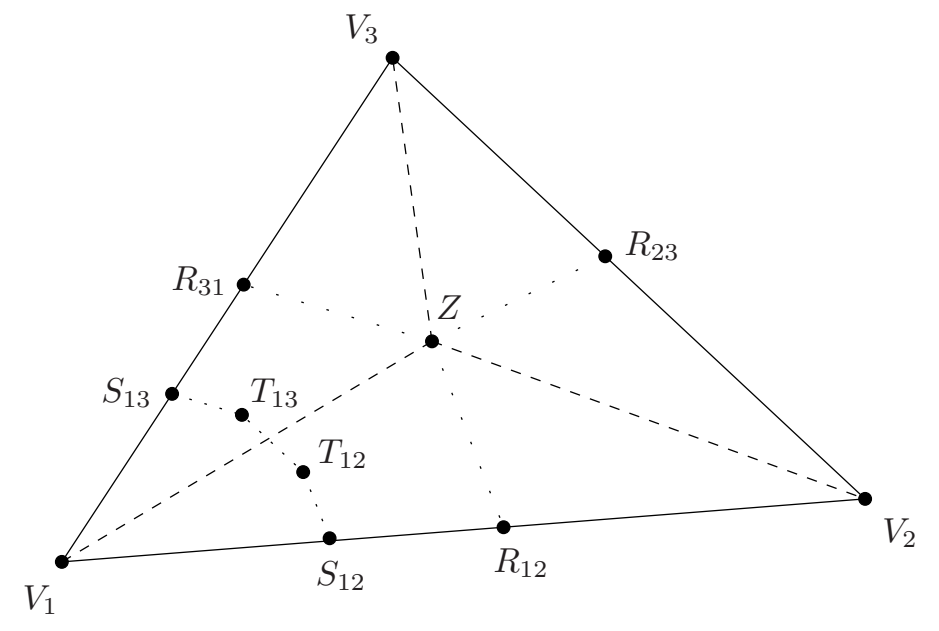

Figure 2: A Clough-Tocher split of a triangle $\mathcal{T}\left(V_{1}, V_{2}, V_{3}\right)$ drawn with dashed lines, and the set of RCT-points $\left(V_{1}, S_{12}, S_{13}, T_{12}, T_{13}\right)$ with respect to vertex $V_{1}$.

By the restriction on the position of the CT-split points, all these barycentric coordinates are nonnegative.

Suppose that the RCT-spline $s(x, y)$ is defined by means of interpolation problem (2.6). On each micro-triangle in $\Delta^{*}$ the spline is a cubic polynomial that can be represented in its Bernstein-Bézier formulation, i.e., with $d=3$ in equations (2.2) and (2.3). The corresponding Bézier ordinates are schematically represented in Figure 3. In view of the $C^{1}$-smoothness at vertex $V_{1}$, the Bézier ordinates in the neighbourhood of $V_{1}$ are found as

$$
\begin{aligned}
s_{1} & =f_{1}, \\
u_{12} & =f_{1}+\frac{1}{3}\left(f_{x, 1}\left(x_{2}-x_{1}\right)+f_{y, 1}\left(y_{2}-y_{1}\right)\right), \\
u_{13} & =f_{1}+\frac{1}{3}\left(f_{x, 1}\left(x_{3}-x_{1}\right)+f_{y, 1}\left(y_{3}-y_{1}\right)\right),
\end{aligned}
$$

and

$$
v_{1}=z_{1} s_{1}+z_{2} u_{12}+z_{3} u_{13}
$$

In a similar way we can compute the Bézier ordinates in the neighbourhood of vertices $V_{2}$ and $V_{3}$. In order to derive the value of $\theta_{3}$, we express the derivative $\frac{\partial s}{\partial \nu_{\varepsilon}}\left(R_{12}\right)$ on edge $\varepsilon=V_{1}-V_{2}$ in terms of the Bézier ordinates of $s(x, y)$, i.e.,

$$
\begin{aligned}
\frac{\partial s}{\partial \nu_{\varepsilon}}\left(R_{12}\right)= & \frac{3}{\left\|Z-R_{12}\right\|}\left(\lambda_{12}^{2}\left(\lambda_{12} s_{1}+\lambda_{21} u_{12}-v_{1}\right)\right. \\
& \left.+2 \lambda_{12} \lambda_{21}\left(\lambda_{12} u_{12}+\lambda_{21} u_{21}-\theta_{3}\right)+\lambda_{21}{ }^{2}\left(\lambda_{12} u_{21}+\lambda_{21} s_{2}-v_{2}\right)\right) .
\end{aligned}
$$

Because the derivative of $s(x, y)$ along edge $\varepsilon$ in the direction $\nu_{\varepsilon}$ is a linear polynomial, we also know that

$$
\frac{\partial s}{\partial \nu_{\varepsilon}}\left(R_{12}\right)=\frac{3}{\left\|Z-R_{12}\right\|}\left(\lambda_{12}\left(\lambda_{12} s_{1}+\lambda_{21} u_{12}-v_{1}\right)+\lambda_{21}\left(\lambda_{12} u_{21}+\lambda_{21} s_{2}-v_{2}\right)\right),
$$




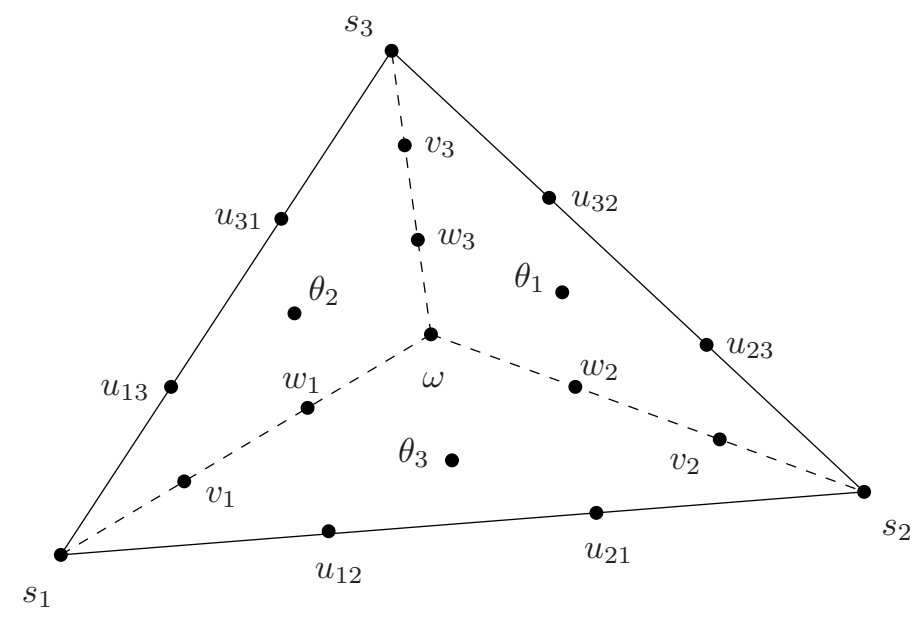

Figure 3: Schematic representation of the Bézier ordinates of an RCT-spline.

and we find

$$
\theta_{3}=\lambda_{12} u_{12}+\lambda_{21} u_{21}+\frac{1}{2}\left(v_{1}+v_{2}-\lambda_{12}\left(s_{1}+u_{21}\right)-\lambda_{21}\left(s_{2}+u_{12}\right)\right) .
$$

We can derive an analogous expression for $\theta_{1}$ and $\theta_{2}$. The remaining Bézier ordinates are obtained from the $C^{1}$-smoothness conditions across the edges in the CT-refinement, i.e.,

$$
\begin{aligned}
w_{1} & =z_{1} v_{1}+z_{2} \theta_{3}+z_{3} \theta_{2}, \\
w_{2} & =z_{1} \theta_{3}+z_{2} v_{2}+z_{3} \theta_{1}, \\
w_{3} & =z_{1} \theta_{2}+z_{2} \theta_{1}+z_{3} v_{3}, \\
\omega & =z_{1} w_{1}+z_{2} w_{2}+z_{3} w_{3} .
\end{aligned}
$$

In this Bernstein-Bézier representation the RCT-splines can be easily represented, evaluated, manipulated and displayed using the de Casteljau algorithm [7].

\section{Normalized reduced Clough-Tocher B-splines}

\subsection{A B-spline representation for RCT-splines}

In this section we look for a suitable representation

$$
s(x, y)=\sum_{i=1}^{n} \sum_{j=1}^{3} c_{i, j} B_{i}^{j}(x, y)
$$

for the splines in $\hat{S}_{3}^{1}\left(\Delta^{*}\right)$. To obtain locally supported basis functions $B_{i}^{j}(x, y)$, we associate with each vertex $V_{i}$ three linearly independent triplets $\left(\alpha_{i, j}, \beta_{i, j}, \gamma_{i, j}\right), j=1,2,3$. The spline $B_{i}^{j}(x, y)$ is defined as the unique solution of interpolation problem (2.6) with all $\left(f_{k}, f_{x, k}, f_{y, k}\right)=(0,0,0)$ except for $k=i$, where $\left(f_{i}, f_{x, i}, f_{y, i}\right)=\left(\alpha_{i, j}, \beta_{i, j}, \gamma_{i, j}\right) \neq(0,0,0)$. Such a spline shall be called a $\mathrm{B}$-spline with respect to vertex $V_{i}$. It is easy to prove that $B_{i}^{j}(x, y)$ vanishes outside the molecule 
of $V_{i}$. The molecule (also called 1-ring) is defined as the union of all triangles in the triangulation that contain $V_{i}$.

We now examine for which choices of $\left(\alpha_{i, j}, \beta_{i, j}, \gamma_{i, j}\right)$ this basis will form a convex partition of unity. From the definition of the B-spline, only three basis functions have a nonzero function and derivative value at vertex $V_{i}$. We then find the necessary and sufficient conditions to form a partition of unity

$$
\begin{aligned}
\alpha_{i, 1}+\alpha_{i, 2}+\alpha_{i, 3} & =1, \\
\beta_{i, 1}+\beta_{i, 2}+\beta_{i, 3} & =0, \\
\gamma_{i, 1}+\gamma_{i, 2}+\gamma_{i, 3} & =0,
\end{aligned}
$$

for $i=1, \ldots, n$. In order to derive conditions ensuring nonnegativity, we consider the B-spline $B_{1}^{j}(x, y)$ corresponding to vertex $V_{1}$. It is sufficient to impose that all Bézier ordinates of the spline are nonnegative. Referring to Figure 3, we know from the definition of the B-spline that the ordinates $s_{2}, s_{3}, u_{21}, u_{23}, u_{32}, u_{31}, v_{2}, v_{3}$ and $\theta_{1}$ are zero. Looking at formulae (2.7)-(2.10), it then suffices to request $s_{1} \geq 0, u_{12} \geq 0, u_{13} \geq 0, \theta_{3} \geq 0$ and $\theta_{2} \geq 0$. This results in the conditions

$$
\begin{aligned}
& \alpha_{1, j} \geq 0, \\
& L_{12, j}:=\alpha_{1, j}+\frac{1}{3}\left(\beta_{1, j}\left(x_{2}-x_{1}\right)+\gamma_{1, j}\left(y_{2}-y_{1}\right)\right) \geq 0, \\
& L_{13, j}:=\alpha_{1, j}+\frac{1}{3}\left(\beta_{1, j}\left(x_{3}-x_{1}\right)+\gamma_{1, j}\left(y_{3}-y_{1}\right)\right) \geq 0, \\
& M_{12, j}:=L_{12, j}+\frac{z_{2}-\lambda_{21}}{6 \lambda_{12}}\left(\beta_{1, j}\left(x_{2}-x_{1}\right)+\gamma_{1, j}\left(y_{2}-y_{1}\right)\right) \\
& +\frac{z_{3}}{6 \lambda_{12}}\left(\beta_{1, j}\left(x_{3}-x_{1}\right)+\gamma_{1, j}\left(y_{3}-y_{1}\right)\right) \geq 0, \\
& M_{13, j}:=L_{13, j}+\frac{z_{2}}{6 \lambda_{13}}\left(\beta_{1, j}\left(x_{2}-x_{1}\right)+\gamma_{1, j}\left(y_{2}-y_{1}\right)\right) \\
& +\frac{z_{3}-\lambda_{31}}{6 \lambda_{13}}\left(\beta_{1, j}\left(x_{3}-x_{1}\right)+\gamma_{1, j}\left(y_{3}-y_{1}\right)\right) \geq 0 .
\end{aligned}
$$

Inequalities (3.3) are also necessary conditions for the nonnegativity of $B_{1}^{j}(x, y)$ on the triangle $\mathcal{T}\left(V_{1}, V_{2}, V_{3}\right)$. Indeed, the B-spline has a negative value at the point $V_{1}$ if $s_{1}<0$. The trace of $B_{1}^{j}(x, y)$ along edge $V_{1}-V_{2}$ is equal to the cubic polynomial $p\left(\tau_{1}\right)=\tau_{1}^{2}\left(\tau_{1} s_{1}+3\left(1-\tau_{1}\right) u_{12}\right)$ with $0 \leq \tau_{1} \leq 1$. It has negative values if $u_{12}<0$. Analogously, the B-spline has negative values along the edges $V_{1}-V_{3}, Z-V_{2}$ and $Z-V_{3}$ if $u_{13}<0, w_{2}=z_{1} \theta_{3}<0$ and $w_{3}=z_{1} \theta_{2}<0$, respectively.

If the molecule of $V_{1}$ has more than one triangle, we have to impose conditions similar to (3.3) for each of these triangles. Since inequality (3.3a) refers to a vertex of $\Delta$, it is sufficient to consider this inequality once. Inequalities (3.3b) and (3.3c) refer to a particular edge of $\Delta$, so they should also be considered only once. Suppose that the molecule has $m$ triangles. In order to ensure the nonnegativity of $B_{1}^{j}(x, y)$, we have to impose in total $3 m+1$ linear constraints if $V_{1}$ is an interior vertex of $\Delta$ and $3 m+2$ linear constraints if $V_{1}$ is a boundary vertex of $\Delta$. Actually, this number of constraints can further be reduced. We do not need to explicitly impose (3.3b) or (3.3c) if they correspond to inner edges of $\Delta$, since then they will be automatically satisfied by the other constraints.

Further on, we will prove that these conditions are always feasible and that there are an infinite number of solutions. 


\subsection{A geometric interpretation}

We now derive a geometric interpretation for the conditions (3.2)-(3.3). For each vertex $V_{i}$ we define three points $Q_{i, j}=\left(X_{i, j}, Y_{i, j}\right), j=1,2,3$, such that

$$
\sum_{i=1}^{n} \sum_{j=1}^{3} X_{i, j} B_{i}^{j}(x, y)=x, \quad \text { and } \sum_{i=1}^{n} \sum_{j=1}^{3} Y_{i, j} B_{i}^{j}(x, y)=y .
$$

Using (3.2) and interpolation problem (2.6), the Cartesian coordinates of $Q_{i, j}$ can be obtained as the solution of the systems

$$
\begin{aligned}
\alpha_{i, 1} X_{i, 1}+\alpha_{i, 2} X_{i, 2}+\alpha_{i, 3} X_{i, 3} & =x_{i} \\
\beta_{i, 1} X_{i, 1}+\beta_{i, 2} X_{i, 2}+\beta_{i, 3} X_{i, 3} & =1 \\
\gamma_{i, 1} X_{i, 1}+\gamma_{i, 2} X_{i, 2}+\gamma_{i, 3} X_{i, 3} & =0
\end{aligned}
$$

and

$$
\begin{aligned}
\alpha_{i, 1} Y_{i, 1}+\alpha_{i, 2} Y_{i, 2}+\alpha_{i, 3} Y_{i, 3} & =y_{i}, \\
\beta_{i, 1} Y_{i, 1}+\beta_{i, 2} Y_{i, 2}+\beta_{i, 3} Y_{i, 3} & =0, \\
\gamma_{i, 1} Y_{i, 1}+\gamma_{i, 2} Y_{i, 2}+\gamma_{i, 3} Y_{i, 3} & =1 .
\end{aligned}
$$

We can compactly write (3.2), (3.5) and (3.6) in the following matrix notation

$$
\left[\begin{array}{ccc}
\alpha_{i, 1} & \alpha_{i, 2} & \alpha_{i, 3} \\
\beta_{i, 1} & \beta_{i, 2} & \beta_{i, 3} \\
\gamma_{i, 1} & \gamma_{i, 2} & \gamma_{i, 3}
\end{array}\right]\left[\begin{array}{ccc}
X_{i, 1} & Y_{i, 1} & 1 \\
X_{i, 2} & Y_{i, 2} & 1 \\
X_{i, 3} & Y_{i, 3} & 1
\end{array}\right]=\left[\begin{array}{ccc}
x_{i} & y_{i} & 1 \\
1 & 0 & 0 \\
0 & 1 & 0
\end{array}\right] .
$$

The triangle $t_{i}\left(Q_{i, 1}, Q_{i, 2}, Q_{i, 2}\right)$ shall be called the RCT-triangle with respect to vertex $V_{i}$.

We will now prove that the constraints (3.3) are equivalent to the request that the following points are inside triangle $t_{1}$ :

$$
\begin{aligned}
V_{1}, & \\
S_{12} & :=\frac{2}{3} V_{1}+\frac{1}{3} V_{2}, \\
S_{13} & :=\frac{2}{3} V_{1}+\frac{1}{3} V_{3}, \\
T_{12} & :=S_{12}+\frac{1}{6 \lambda_{12}}\left(Z-R_{12}\right), \\
T_{13} & :=S_{13}+\frac{1}{6 \lambda_{13}}\left(Z-R_{13}\right) .
\end{aligned}
$$

These points shall be called RCT-points with respect to vertex $V_{1}$. They are depicted in Figure 2 . Following a similar idea as the one used in [4] for Powell-Sabin B-splines, we compute the barycentric coordinates $\left(\sigma_{1}, \sigma_{2}, \sigma_{3}\right)$ of these points with respect to RCT-triangle $t_{1}$ and impose them to be nonnegative. Let $\left(\tau_{1}, \tau_{2}, \tau_{3}\right)$ be the barycentric coordinates of the same points with respect to triangle $\mathcal{T}\left(V_{1}, V_{2}, V_{3}\right)$. Then, by (2.1), it holds that

$$
\left[\begin{array}{l}
\sigma_{1} \\
\sigma_{2} \\
\sigma_{3}
\end{array}\right]=A\left[\begin{array}{l}
\tau_{1} \\
\tau_{2} \\
\tau_{3}
\end{array}\right]
$$

with

$$
A=\left[\begin{array}{ccc}
X_{1,1} & X_{1,2} & X_{1,3} \\
Y_{1,1} & Y_{1,2} & Y_{1,3} \\
1 & 1 & 1
\end{array}\right]^{-1}\left[\begin{array}{ccc}
x_{1} & x_{2} & x_{3} \\
y_{1} & y_{2} & y_{3} \\
1 & 1 & 1
\end{array}\right]
$$


Using (3.7) we find that

$$
\begin{aligned}
A & =\left[\begin{array}{lll}
\alpha_{1,1} & \beta_{1,1} & \gamma_{1,1} \\
\alpha_{1,2} & \beta_{1,2} & \gamma_{1,2} \\
\alpha_{1,3} & \beta_{1,3} & \gamma_{1,3}
\end{array}\right]\left[\begin{array}{ccc}
x_{1} & 1 & 0 \\
y_{1} & 0 & 1 \\
1 & 0 & 0
\end{array}\right]^{-1}\left[\begin{array}{ccc}
x_{1} & x_{2} & x_{3} \\
y_{1} & y_{2} & y_{3} \\
1 & 1 & 1
\end{array}\right] \\
& =\left[\begin{array}{lll}
\alpha_{1,1} & \alpha_{1,1}+\beta_{1,1}\left(x_{2}-x_{1}\right)+\gamma_{1,1}\left(y_{2}-y_{1}\right) & \alpha_{1,1}+\beta_{1,1}\left(x_{3}-x_{1}\right)+\gamma_{1,1}\left(y_{3}-y_{1}\right) \\
\alpha_{1,2} & \alpha_{1,2}+\beta_{1,2}\left(x_{2}-x_{1}\right)+\gamma_{1,2}\left(y_{2}-y_{1}\right) & \alpha_{1,2}+\beta_{1,2}\left(x_{3}-x_{1}\right)+\gamma_{1,2}\left(y_{3}-y_{1}\right) \\
\alpha_{1,3} & \alpha_{1,3}+\beta_{1,3}\left(x_{2}-x_{1}\right)+\gamma_{1,3}\left(y_{2}-y_{1}\right) & \alpha_{1,3}+\beta_{1,3}\left(x_{3}-x_{1}\right)+\gamma_{1,3}\left(y_{3}-y_{1}\right)
\end{array}\right] .
\end{aligned}
$$

The barycentric coordinates $\left(\tau_{1}, \tau_{2}, \tau_{3}\right)$ of the RCT-points with respect to $\mathcal{T}\left(V_{1}, V_{2}, V_{3}\right)$ are

$$
\begin{aligned}
& V_{1}=(1,0,0), \quad S_{12}=\left(\frac{2}{3}, \frac{1}{3}, 0\right), \quad S_{13}=\left(\frac{2}{3}, 0, \frac{1}{3}\right), \\
& T_{12}=\left(\frac{2}{3}+\frac{z_{1}-\lambda_{12}}{6 \lambda_{12}}, \frac{1}{3}+\frac{z_{2}-\lambda_{21}}{6 \lambda_{12}}, \frac{z_{3}}{6 \lambda_{12}}\right), \\
& T_{13}=\left(\frac{2}{3}+\frac{z_{1}-\lambda_{13}}{6 \lambda_{13}}, \frac{z_{2}}{6 \lambda_{13}}, \frac{1}{3}+\frac{z_{3}-\lambda_{31}}{6 \lambda_{13}}\right) .
\end{aligned}
$$

Substituting these values into (3.9), we get the following barycentric coordinates $\left(\sigma_{1}, \sigma_{2}, \sigma_{3}\right)$ with respect to $\mathrm{RCT}$-triangle $t_{1}$ :

$$
\begin{aligned}
& V_{1}=\left(\alpha_{1,1}, \alpha_{1,2}, \alpha_{1,3}\right), \quad S_{12}=\left(L_{12,1}, L_{12,2}, L_{12,3}\right), \quad S_{13}=\left(L_{13,1}, L_{13,2}, L_{13,3}\right), \\
& T_{12}=\left(M_{12,1}, M_{12,2}, M_{12,3}\right), \quad T_{13}=\left(M_{13,1}, M_{13,2}, M_{13,3}\right) .
\end{aligned}
$$

Imposing that these coordinates are nonnegative corresponds to the constraints (3.3).

We can interpret our problem as finding a triangle that must contain a number of specified points. It is clear that this geometric problem always has a solution. From a stability point of view it is preferred to select a small triangle. An appropriate choice, as suggested in [4] for PS-triangles, is to calculate triangles of minimal area, the so-called optimal triangles. Computationally, this problem leads to a quadratic programming problem. Since by (3.7)

$$
\left|\begin{array}{ccc}
X_{i, 1} & Y_{i, 1} & 1 \\
X_{i, 2} & Y_{i, 2} & 1 \\
X_{i, 3} & Y_{i, 3} & 1
\end{array}\right|=\left|\begin{array}{ccc}
\alpha_{i, 1} & \alpha_{i, 2} & \alpha_{i, 3} \\
\beta_{i, 1} & \beta_{i, 2} & \beta_{i, 3} \\
\gamma_{i, 1} & \gamma_{i, 2} & \gamma_{i, 3}
\end{array}\right|^{-1}=\frac{1}{\beta_{i, 1} \gamma_{i, 2}-\gamma_{i, 1} \beta_{i, 2}}
$$

the construction of an optimal RCT-triangle $t_{i}$ is equivalent to the quadratic programming problem

$$
\max \left(\beta_{i, 1} \gamma_{i, 2}-\gamma_{i, 1} \beta_{i, 2}\right),
$$

subjected to the linear constraints (3.2)-(3.3). Since we are looking for a maximum, we will always obtain a positive value of the objective function as result. Suppose that $\beta_{i, 1} \gamma_{i, 2}-\gamma_{i, 1} \beta_{i, 2}<0$, then there exists another set of triplets $\left(\hat{\alpha}_{i, j}, \hat{\beta}_{i, j}, \hat{\gamma}_{i, j}\right), j=1,2,3$, with $\hat{\alpha}_{i, 1}=\alpha_{i, 2}, \hat{\alpha}_{i, 2}=\alpha_{i, 1}$, $\hat{\alpha}_{i, 3}=\alpha_{i, 3}, \hat{\beta}_{i, 1}=\beta_{i, 2}, \hat{\beta}_{i, 2}=\beta_{i, 1}, \hat{\beta}_{i, 3}=\beta_{i, 3}, \hat{\gamma}_{i, 1}=\gamma_{i, 2}, \hat{\gamma}_{i, 2}=\gamma_{i, 1}$ and $\hat{\gamma}_{i, 3}=\gamma_{i, 3}$. They also satisfy (3.2)-(3.3) and it holds that $\hat{\beta}_{i, 1} \hat{\gamma}_{i, 2}-\hat{\gamma}_{i, 1} \hat{\beta}_{i, 2}>0$.

In Figure 4 we illustrate the RCT-points and a set of optimal RCT-triangles for a triangulation taken from [5]. Figure 5 shows the same triangulation with a set of optimal PS-triangles. In this example the RCT-triangles are on average 1.7 times larger than the corresponding PS-triangles.

Finally, we briefly consider the inverse problem. Given the position of the points $Q_{i, j}$, the triplets $\left(\alpha_{i, j}, \beta_{i, j}, \gamma_{i, j}\right)$ can be computed as follows. We already pointed out that $\left(\alpha_{i, 1}, \alpha_{i, 2}, \alpha_{i, 3}\right)$ can be 


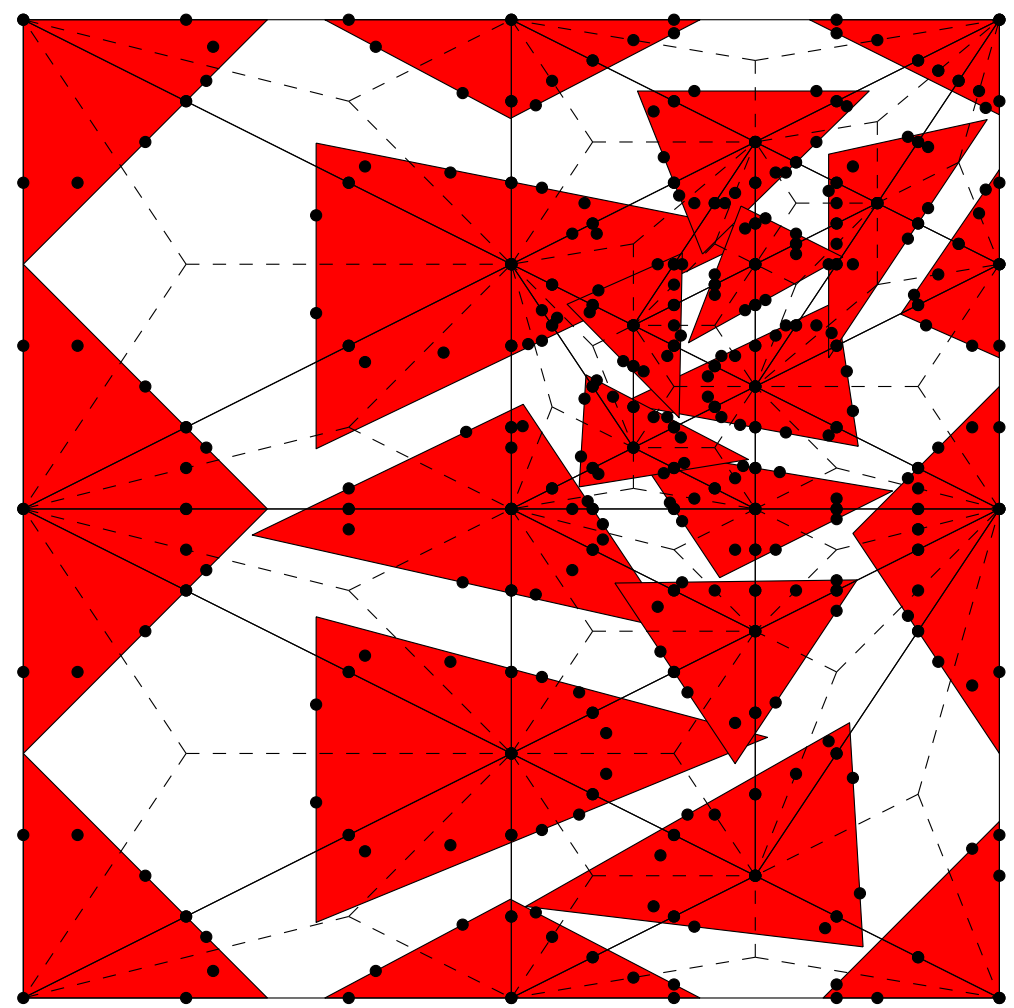

Figure 4: A triangulation with a set of optimal RCT-triangles.

interpreted as the barycentric coordinates of vertex $V_{i}$ with respect to $t_{i}\left(Q_{i, 1}, Q_{i, 2}, Q_{i, 2}\right)$. From (3.7) we obtain that

$$
\begin{gathered}
\beta_{i, 1}=\frac{Y_{i, 2}-Y_{i, 3}}{E}, \quad \beta_{i, 2}=\frac{Y_{i, 3}-Y_{i, 1}}{E}, \quad \beta_{i, 3}=\frac{Y_{i, 1}-Y_{i, 2}}{E}, \\
\gamma_{i, 1}=\frac{X_{i, 3}-X_{i, 2}}{E}, \quad \gamma_{i, 2}=\frac{X_{i, 1}-X_{i, 3}}{E}, \quad \gamma_{i, 3}=\frac{X_{i, 2}-X_{i, 1}}{E},
\end{gathered}
$$

with

$$
E=\left|\begin{array}{ccc}
X_{i, 1} & Y_{i, 1} & 1 \\
X_{i, 2} & Y_{i, 2} & 1 \\
X_{i, 3} & Y_{i, 3} & 1
\end{array}\right| .
$$

The triplets $\left(\beta_{i, 1}, \beta_{i, 2}, \beta_{i, 3}\right)$ and $\left(\gamma_{i, 1}, \gamma_{i, 2}, \gamma_{i, 3}\right)$ can be seen as the barycentric coordinates of the $x$ and $y$-direction with respect to $t_{i}$.

\section{Applications}

\subsection{Control triangles of an RCT-spline}

Referring to the RCT-spline representation (3.1) and the definition of the RCT-triangles (3.4), we define control points as

$$
\mathbf{c}_{i, j}=\left(X_{i, j}, Y_{i, j}, c_{i, j}\right),
$$




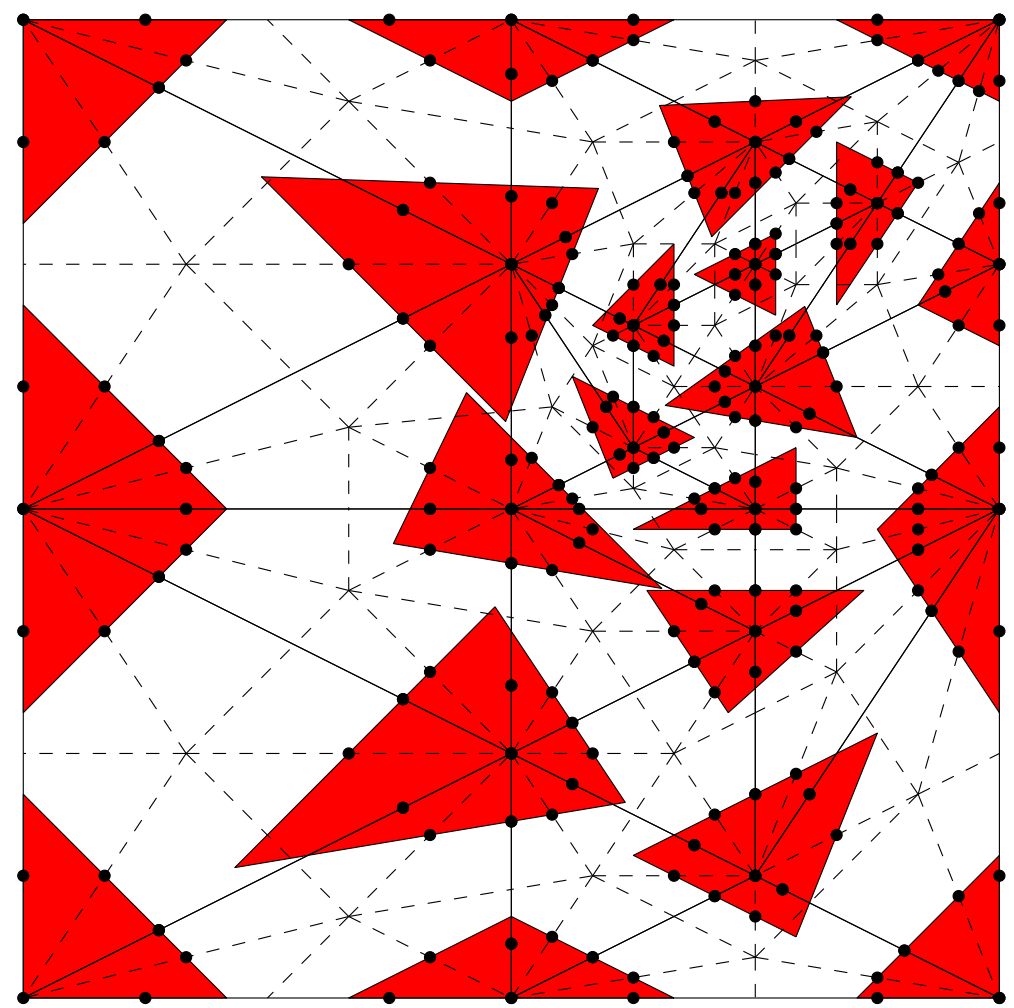

Figure 5: A triangulation with a set of optimal PS-triangles.

with $i=1, \ldots, n$ and $j=1,2,3$. Since the RCT-spline basis forms a convex partition of unity, it follows that the graph of spline (3.1) lies inside the convex hull of the control points (4.1). These points can be considered as vertices of the triangles $T_{i}\left(\mathbf{c}_{i, 1}, \mathbf{c}_{i, 2}, \mathbf{c}_{i, 3}\right), i=1, \ldots, n$, which we shall call control triangles.

By the definition of the B-splines we know that

$$
\begin{aligned}
s\left(V_{i}\right) & =\alpha_{i, 1} c_{i, 1}+\alpha_{i, 2} c_{i, 2}+\alpha_{i, 3} c_{i, 3}, \\
\frac{\partial s}{\partial x}\left(V_{i}\right) & =\beta_{i, 1} c_{i, 1}+\beta_{i, 2} c_{i, 2}+\beta_{i, 3} c_{i, 3}, \\
\frac{\partial s}{\partial y}\left(V_{i}\right) & =\gamma_{i, 1} c_{i, 1}+\gamma_{i, 2} c_{i, 2}+\gamma_{i, 3} c_{i, 3} .
\end{aligned}
$$

Inverting the system (4.2), and using (3.7), we find after some elementary calculations that

$$
\begin{aligned}
& c_{i, 1}=s\left(V_{i}\right)+\left(X_{i, 1}-x_{i}\right) \frac{\partial s}{\partial x}\left(V_{i}\right)+\left(Y_{i, 1}-y_{i}\right) \frac{\partial s}{\partial y}\left(V_{i}\right), \\
& c_{i, 2}=s\left(V_{i}\right)+\left(X_{i, 2}-x_{i}\right) \frac{\partial s}{\partial x}\left(V_{i}\right)+\left(Y_{i, 2}-y_{i}\right) \frac{\partial s}{\partial y}\left(V_{i}\right), \\
& c_{i, 3}=s\left(V_{i}\right)+\left(X_{i, 3}-x_{i}\right) \frac{\partial s}{\partial x}\left(V_{i}\right)+\left(Y_{i, 3}-y_{i}\right) \frac{\partial s}{\partial y}\left(V_{i}\right) .
\end{aligned}
$$

It follows that the three control points $\mathbf{c}_{i, j}, j=1,2,3$, belong to the plane tangent to the spline surface $z=s(x, y)$ at vertex $V_{i}$. Thus, the control triangle $T_{i}$ is tangent to the spline surface at $V_{i}$. 

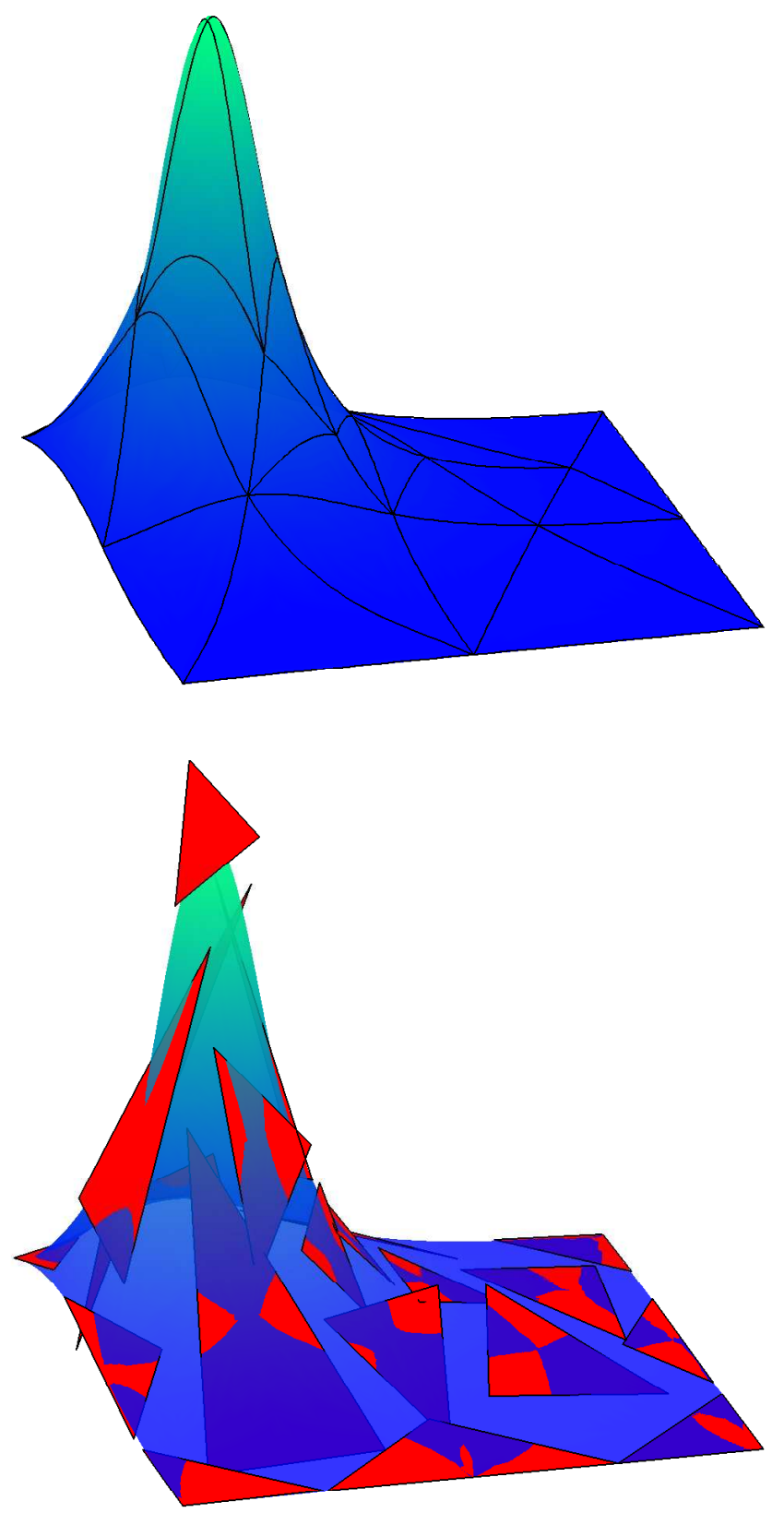

Figure 6: An RCT-spline surface with its control triangles corresponding to the triangulation shown in Figure 4.

Using these control triangles one can interactively change the shape of the spline surface in a predictable way. Because of the local support of the B-splines, a change of control triangle $T_{i}$ will only affect the spline patches related to the triangles in the molecule of vertex $V_{i}$. 
Figure 6 shows an RCT-spline surface together with its control triangles. The spline is the Hermite interpolant of the function $f(x, y)=\left(\exp \left((x-0.52)^{2}+(y-0.48)^{2}\right)-0.95\right)^{-1}$ on domain $\Omega=$ $[-1,1] \times[-1,1]$, where the data points are located at the vertices of the triangulation in Figure 4.

\subsection{Bézier ordinates of an RCT-spline}

The Bézier ordinates of an RCT-spline in the representation (3.1) can be computed in a stable way from its B-spline coefficients $c_{i, j}$. We consider again the triangle $\mathcal{T}\left(V_{1}, V_{2}, V_{3}\right)$ in Figure 2 and the Bézier ordinates in Figure 3.

Combining expressions (2.7), (3.3b)-(3.3c) and (4.2), we derive that the Bézier ordinates in the neighbourhood of vertex $V_{1}$ only depend on the three coefficients $c_{1, j}$ with $j=1,2,3$ :

$$
\begin{aligned}
s_{1} & =\alpha_{1,1} c_{1,1}+\alpha_{1,2} c_{1,2}+\alpha_{1,3} c_{1,3}, \\
u_{12} & =L_{12,1} c_{1,1}+L_{12,2} c_{1,2}+L_{12,3} c_{1,3}, \\
u_{13} & =L_{13,1} c_{1,1}+L_{13,2} c_{1,2}+L_{13,3} c_{1,3} .
\end{aligned}
$$

They are convex combinations since the weights are the barycentric coordinates of the RCT-points $V_{1}, S_{12}$ and $S_{13}$ with respect to RCT-triangle $t_{1}\left(Q_{1,1}, Q_{1,2}, Q_{1,3}\right)$. In a similar way we can compute $\left(s_{2}, u_{23}, u_{21}\right)$ and $\left(s_{3}, u_{31}, u_{32}\right)$ from the B-spline coefficients $c_{2, j}$ and $c_{3, j}$ respectively. Using (2.9), (3.3d) and (4.2), we find that

$$
\theta_{3}=\lambda_{12}\left(M_{12,1} c_{1,1}+M_{12,2} c_{1,2}+M_{12,3} c_{1,3}\right)+\lambda_{21}\left(M_{21,1} c_{2,1}+M_{21,2} c_{2,2}+M_{21,3} c_{2,3}\right),
$$

with $\left(M_{12,1}, M_{12,2}, M_{12,3}\right)$ and $\left(M_{21,1}, M_{21,2}, M_{21,3}\right)$ the barycentric coordinates of the RCT-points $T_{12}$ and $T_{21}$ with respect to RCT-triangles $t_{1}\left(Q_{1,1}, Q_{1,2}, Q_{1,3}\right)$ and $t_{2}\left(Q_{2,1}, Q_{2,2}, Q_{2,3}\right)$ respectively. Note that

$$
\lambda_{12} T_{12}+\lambda_{21} T_{21}=\frac{1}{3}\left(V_{1}+V_{2}+Z\right) .
$$

This can be verified using definition (3.8d). Expressions similar to (4.5) hold for $\theta_{1}$ and $\theta_{2}$. The remaining Bézier ordinates are obtained by the formulae given in (2.8) and (2.10).

Since only convex combinations are needed in the computation of these Bézier ordinates and in the de Casteljau algorithm, we obtain a stable algorithm for the evaluation of an RCT-spline in its normalized B-spline representation.

More general, if we apply the convex combinations (4.4), (4.5), (2.8) and (2.10) to the control points $\left(X_{i, j}, Y_{i, j}, c_{i, j}\right)$, we directly get the Bézier control points of the RCT-spline surface. Figure 7 shows the Bézier control net of the RCT-spline depicted in Figure 6.

\subsection{Parametric RCT-spline surfaces}

A parametric RCT-spline surface is defined as

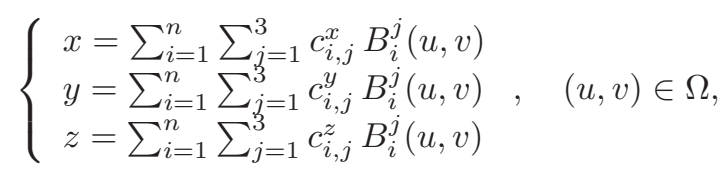

or, compactly,

$$
\mathbf{s}(u, v)=\sum_{i=1}^{n} \sum_{j=1}^{3} \mathbf{c}_{i, j} B_{i}^{j}(u, v)
$$




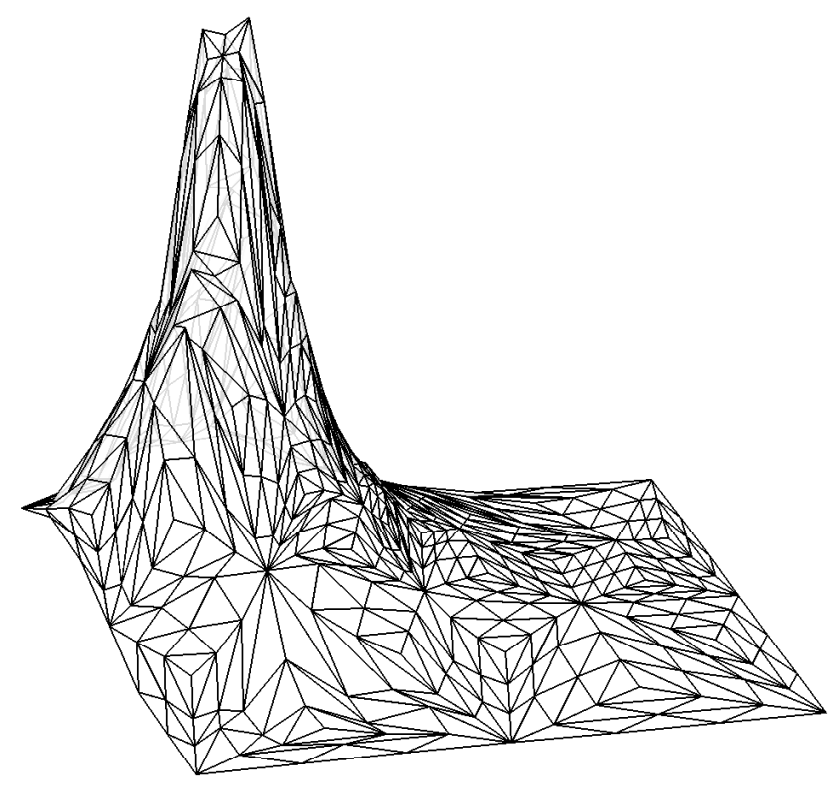

Figure 7: Bézier control net of an RCT-spline surface.

where the $\mathbf{c}_{i, j}=\left(c_{i, j}^{x}, c_{i, j}^{y}, c_{i, j}^{z}\right)$ are again called control points. Note that in the parametric setting the choice of the control points $\mathbf{c}_{i, j}$ is completely free, whereas for RCT-splines only the $z$-component of the control points can be chosen. Referring to (3.1), the graph of an RCT-spline is a particular case of the parametric RCT-spline surface, where $x=u$ and $y=v$ (i.e., $c_{i, j}^{x}=X_{i, j}$ and $c_{i, j}^{y}=Y_{i, j}$ ).

A parametric surface $\mathbf{s}(u, v)$ lies within the convex hull of its control points. We can associate a control triangle $T_{i}\left(\mathbf{c}_{i, 1}, \mathbf{c}_{i, 2}, \mathbf{c}_{i, 3}\right)$ with each vertex $V_{i}$ in the parameter domain. Referring to the locality of the B-splines, it is easy to verify that the (component-wise) evaluation of $\mathbf{s}(u, v)$ and its partial derivatives at vertex $V_{i}$ yields

$$
\begin{aligned}
\mathbf{s}\left(V_{i}\right) & =\alpha_{i, 1} \mathbf{c}_{i, 1}+\alpha_{i, 2} \mathbf{c}_{i, 2}+\alpha_{i, 3} \mathbf{c}_{i, 3}, \\
\frac{\partial \mathbf{s}}{\partial u}\left(V_{i}\right) & =\beta_{i, 1} \mathbf{c}_{i, 1}+\beta_{i, 2} \mathbf{c}_{i, 2}+\beta_{i, 3} \mathbf{c}_{i, 3}, \\
\frac{\partial \mathbf{s}}{\partial v}\left(V_{i}\right) & =\gamma_{i, 1} \mathbf{c}_{i, 1}+\gamma_{i, 2} \mathbf{c}_{i, 2}+\gamma_{i, 3} \mathbf{c}_{i, 3} .
\end{aligned}
$$

It follows that the control triangle is tangent to the surface at $\mathbf{s}\left(V_{i}\right)$. The normal vector $\mathbf{n}$ to the surface at $\mathbf{s}\left(V_{i}\right)$ can be computed as

$$
\mathbf{n}=\frac{\partial \mathbf{s}}{\partial u}\left(V_{i}\right) \times \frac{\partial \mathbf{s}}{\partial v}\left(V_{i}\right) .
$$

By (3.2), (3.12)-(3.13) and (4.9) we get

$$
\begin{aligned}
\mathbf{n} & =\left(\beta_{i, 1} \mathbf{c}_{i, 1}+\beta_{i, 2} \mathbf{c}_{i, 2}+\beta_{i, 3} \mathbf{c}_{i, 3}\right) \times\left(\gamma_{i, 1} \mathbf{c}_{i, 1}+\gamma_{i, 2} \mathbf{c}_{i, 2}+\gamma_{i, 3} \mathbf{c}_{i, 3}\right) \\
& =\left(\beta_{i, 1} \gamma_{i, 2}-\gamma_{i, 1} \beta_{i, 2}\right)\left(\left(\mathbf{c}_{i, 1}-\mathbf{c}_{i, 3}\right) \times\left(\mathbf{c}_{i, 2}-\mathbf{c}_{i, 3}\right)\right) \\
& =\frac{1}{E}\left(\left(\mathbf{c}_{i, 1}-\mathbf{c}_{i, 3}\right) \times\left(\mathbf{c}_{i, 2}-\mathbf{c}_{i, 3}\right)\right) .
\end{aligned}
$$




\section{Concluding remarks}

In this paper we presented a suitable normalized B-spline representation for reduced Clough-Tocher splines. The basis functions have a local support, they are nonnegative, and they form a partition of unity. We derived a set of necessary and sufficient conditions on the triplets $\left(\alpha_{i, j}, \beta_{i, j}, \gamma_{i, j}\right)$, $j=1,2,3$, which define the three B-splines associated with vertex $V_{i}$. The construction has a geometrical interpretation. The problem is equivalent to the determination of an RCT-triangle $t_{i}$ that must contain a specific set of points. The triplets $\left(\alpha_{i, j}, \beta_{i, j}, \gamma_{i, j}\right)$ are related to the vertices $Q_{i, j}$ of this triangle.

We considered a number of applications useful in computer aided geometric design. The normalized basis allows the definition of control points and control triangles. They can be used to interactively change the shape of the spline in a predictable way. We showed how to compute from the control points of an RCT-spline its corresponding Bézier control net in a stable way. An extension to more general parametric RCT-spline surfaces is also straightforward.

Finally, we discuss related spline functions found in the literature. In [2] a slightly different cubic spline space is described. The normal derivative of such a spline along an edge is restricted to be a linear polynomial. It is possible to adopt the proposed construction of a normalized basis for this related spline space, on condition that the position of the split points in the CT-refinement is restricted in a particular way. For each triangle $\mathcal{T}$ and each edge $\varepsilon$ of $\mathcal{T}$, the orthogonal projection of the split point of $\mathcal{T}$ onto $\varepsilon$ must lie on $\varepsilon$. The optimization strategy in [12] to obtain rangerestricted CT-splines requires the same restriction on the split points. This restriction is stronger than the one assumed in Section 2.2 for our RCT-spline space.

\section{Acknowledgement}

Hendrik Speleers is a Postdoctoral Fellow of the Research Foundation Flanders (Belgium).

\section{References}

[1] M. Bernadou and K. Hassan. Basis functions for general Hsieh-Clough-Tocher triangles, complete or reduced. Int. J. Numer. Meth. Engrg., 17:784-789, 1981.

[2] P.G. Ciarlet. Interpolation error estimates for the reduced Hsieh-Clough-Tocher triangle. Math. Comp., 32:335-344, 1978.

[3] R.W. Clough and J.L. Tocher. Finite element stiffness matrices for analysis of plates in bending. In Conf. on Matrix Methods in Structural Mechanics, pages 515-545, Wright Patterson Air Force Base, Ohio, 1965.

[4] P. Dierckx. On calculating normalized Powell-Sabin B-splines. Comput. Aided Geom. Design, 15:61-78, 1997.

[5] P. Dierckx, S. Van Leemput, and T. Vermeire. Algorithms for surface fitting using PowellSabin splines. IMA J. Numer. Anal., 12:271-299, 1992.

[6] G. Farin. A modified Clough-Tocher interpolant. Comput. Aided Geom. Design, 2:19-27, 1985.

[7] G. Farin. Triangular Bernstein-Bézier patches. Comput. Aided Geom. Design, 3:83-127, 1986. 
[8] G. Farin. Curves and Surfaces for CAGD: A Practical Guide. Morgan Kaufmann Publishers, San Francisco, fifth edition, 2002.

[9] J. Maes and A. Bultheel. Stable multiresolution analysis on triangles for surface compression. Electr. Trans. Numer. Anal., 25:224-258, 2006.

[10] S. Mann. Cubic precision Clough-Tocher interpolation. Comput. Aided Geom. Design, 16:8588, 1999.

[11] C. Manni and P. Sablonnière. Quadratic spline quasi-interpolants on Powell-Sabin partitions. Adv. Comput. Math., 26:283-304, 2007.

[12] J.W. Schmidt. Range restricted interpolation by cubic $C^{1}$-splines on Clough-Tocher splits. In W. Haußmann, K. Jetter, and M. Reimer, editors, Advances in Multivariate Approximation, pages 253-267. Wiley-VCH, Berlin, 1999.

[13] H. Speleers, P. Dierckx, and S. Vandewalle. Numerical solution of partial differential equations with Powell-Sabin splines. J. Comput. Appl. Math., 189:643-659, 2006.

[14] H. Speleers, P. Dierckx, and S. Vandewalle. Quasi-hierarchical Powell-Sabin B-splines. Comput. Aided Geom. Design, 26:174-191, 2009. 